\title{
The synthesis of L-gulose and L-xylose from D-gluconolactone
}

\author{
Wen-Bin Yang, ${ }^{a}$ Sachindra S. Patil, ${ }^{a}$ Cheng-Hsin Tsai, ${ }^{a, b}$ Chun-Hung Lin ${ }^{a}$,* and Jim-Min Fang ${ }^{b}$ \\ ${ }^{a}$ Institute of Biological Chemistry, Academia Sinica, No. 128, Academia Road Section II, Nan-Kang, Taipei 11529-46, Taiwan \\ ${ }^{\mathrm{b}}$ Department of Chemistry, National Taiwan University, Taipei, Taiwan
}

Received 14 August 2001; accepted 16 November 2001

\begin{abstract}
L-Gulose, an essential component for the antibiotic and antitumor activities of bleomycin $\mathrm{A}_{2}$, was synthesized in $47 \%$ yield from D-glucono-1,5-lactone. The effective cleavage of acetonides by $\mathrm{SnCl}_{2}$ in the presence of tert-butyldimethylsilyl ethers was of importance in the course of synthesis. Elaboration of D-glucono-1,5-lactone that includes a step of oxidative degradation by Dess-Martin periodinane also afforded a respectable yield of L-xylose. (C) 2002 Elsevier Science Ltd. All rights reserved.
\end{abstract}

\section{Introduction}

The use of aldonolactones in the preparation of biologically interesting molecules, such as $C$-glycosides, carbasugars and azasugars, has been documented in several reports. ${ }^{1}$ These studies mainly focus on the manipulation of lactone functionality. ${ }^{2,3}$ In comparison with common sugars (e.g. glucose and galactose), sugar lactones are seldom used directly as the starting materials in organic syntheses, though many of them are commercially available at low cost. This consequence may be partly due to the low $\mathrm{p} K_{\mathrm{a}}$ of their C-2 hydrogens that often cause problems of elimination under basic conditions. The preference for the formation of 1,4-lactones over 1,5-lactones may also limit their synthetic applications. ${ }^{4}$ As a continuation of our study of sugar lactones, we demonstrate herein the synthesis of L-xylose and L-gulose (less available in nature) from a common precursor, D-gluconolactone. A Lewis acid $\mathrm{SnCl}_{2}$ is found to be an especially effective promoter in the protection of diol functionalities, and in the deprotection of the acetonides.

\section{Results and discussion}

Chittenden has reported a two-step synthesis of acetonide 2 (less than $40 \%$ yield) from D-glucono-1,5-lactone (1) via the intermediacy of D-glucono-1,4-lactone. ${ }^{5}$ We devised an improved procedure ${ }^{6}$ for the direct formation of acetonide 2 as shown in Scheme 1. In the presence of $\mathrm{SnCl}_{2}$, the sugar lactone 1 in DMF solution was treated with 2,2-dimethoxypropane at $40^{\circ} \mathrm{C}$ for $15 \mathrm{~h}$. After DMF and excess 2,2-dimethoxypropane were removed, acetonide 2 was obtained

\footnotetext{
Keywords: L-gulose; L-xylose; lactone; oxidation; tin(II) chloride.

* Corresponding author. Tel.: +886-2-2789-0110; fax: +886-2-2651-

4705; e-mail: chunhung@gate.sinica.edu.tw
}

in $85 \%$ yield by chromatography on a silica gel column. In order to ensure a high and reproducible yield, the acetalization should be conducted under anhydrous conditions, and prolonged chromatography should be avoided.

In case the moisture was not rigorously excluded, two diacetonide esters $\mathbf{7 a}$ and $\mathbf{7 b}$ (Fig. 1) were also obtained as the side products. When D-glucono-1,5-lactone was treated with acetone in the presence of iodine, diacetonide $\mathbf{7 c}$ and triacetonide $\mathbf{7 d}$ were obtained instead of the desired
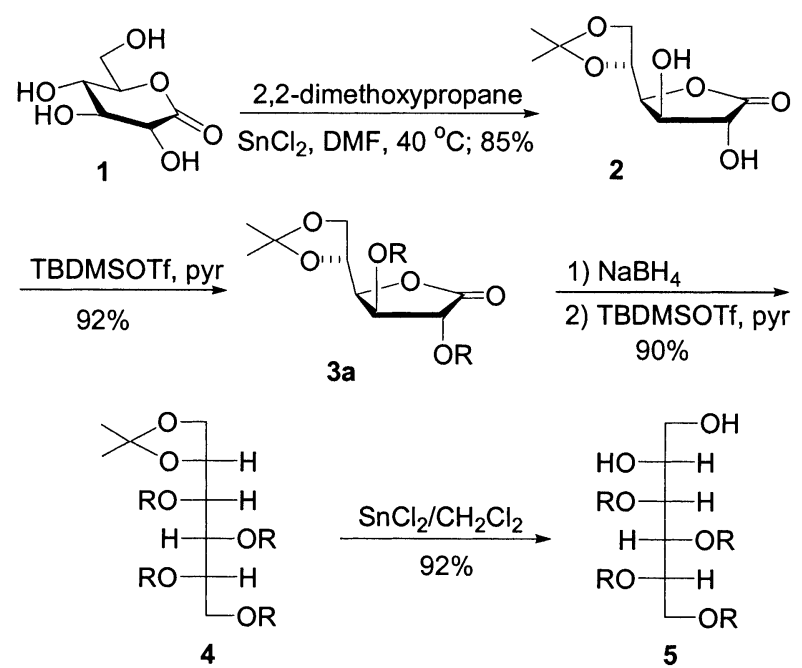

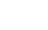
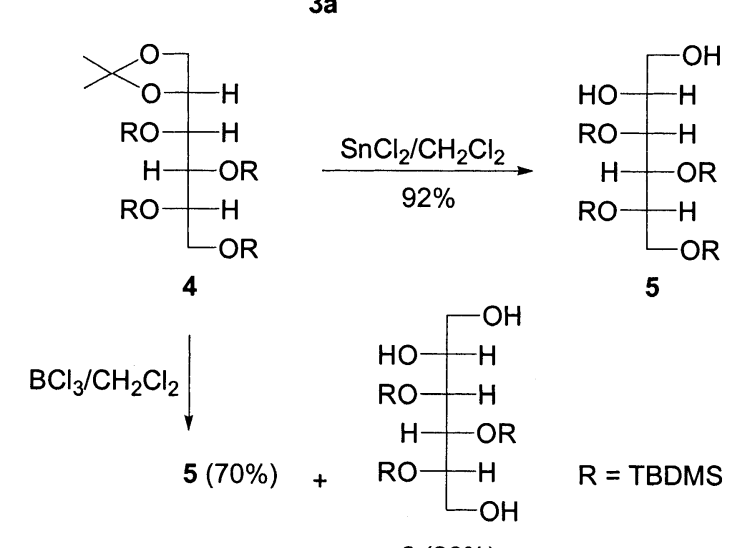

$R=T B D M S$ 
<smiles>COC(=O)[C@H]1O[C@@H](C(O)[C@H]2COC(C)(C)O2)[C@@H](O)C1(C)C</smiles><smiles>COC(=O)C(O)[C@@H]1CC(C)(C)O[C@H]([C@@H]2COC(C)(C)O2)O1</smiles><smiles>CC1(C)OC[C@@H]([C@H](O)[C@H](O)C(=O)O)[C@@H]1O</smiles><smiles>CC1(C)OC[C@@H]([C@H](O)[C@H]2OC(C)(C)OC2=O)O1</smiles>

Figure 1.

acetonide 2 . The structures of $7 \mathbf{a}-\mathbf{d}$ were determined by comparison of their ${ }^{1} \mathrm{H}$ and ${ }^{13} \mathrm{C}$ NMR spectra with those reported in literature. ${ }^{7}$ The structure of $7 \mathbf{c}$ was confirmed by an X-ray diffraction analysis, as shown in Fig. 2.
Acetonide 2 was subjected to silylation with tert-butyldimethylsilyl (trifluoromethane)sulfonate (TBDMSOTf), giving lactone $\mathbf{3 a}$ in $92 \%$ yield. Reduction of lactone 3a with $\mathrm{NaBH}_{4}$, followed by silylation, afforded compound 4 in $90 \%$ yield. Deprotection of the acetonide moiety was achieved in a chemoselective manner by using $\mathrm{SnCl}_{2}$ as the promoter to give diol $\mathbf{5}$ in $92 \%$ yield. When $\mathrm{BCl}_{3}$ was used to replace $\mathrm{SnCl}_{2}$, the reaction gave diol $5(70 \%)$ and a side product of triol $\mathbf{6}(20 \%)$ derived from a concurrent cleavage of the primary silyl ether. ${ }^{6 \mathrm{~b}}$ The beneficial effect of $\mathrm{SnCl}_{2}$ was also applicable to the related reactions (Fig. 3). The acetonide moieties in compounds $\mathbf{3 b}$ and 9 were removed without interference with the silyl and ester groups (OTBDMS and $\mathrm{CO}_{2} \mathrm{Me}$ ). A selective cleavage of the less hindered acetonide of 1,2:5,6- $O$-isopropylidene- $\alpha$-D-glucofuranose (11) was also realized by treatment with $\mathrm{SnCl}_{2}$ in $\mathrm{CH}_{2} \mathrm{Cl}_{2}$ solution.

At this stage, compound $\mathbf{5}$ played a pivotal role in the synthesis of L-xylose and L-gulose (Scheme 2). The vicinal diol underwent an oxidative degradation on treatment with Dess-Martin periodinane ${ }^{8}$ to give aldehyde 13 in $73 \%$

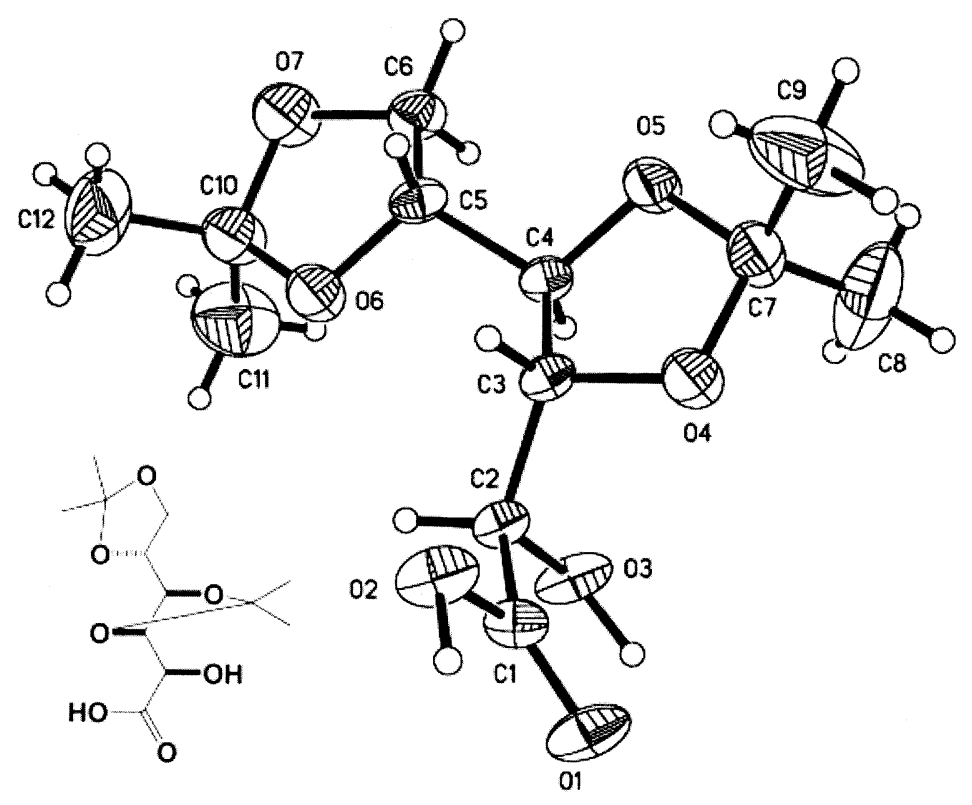

Figure 2.

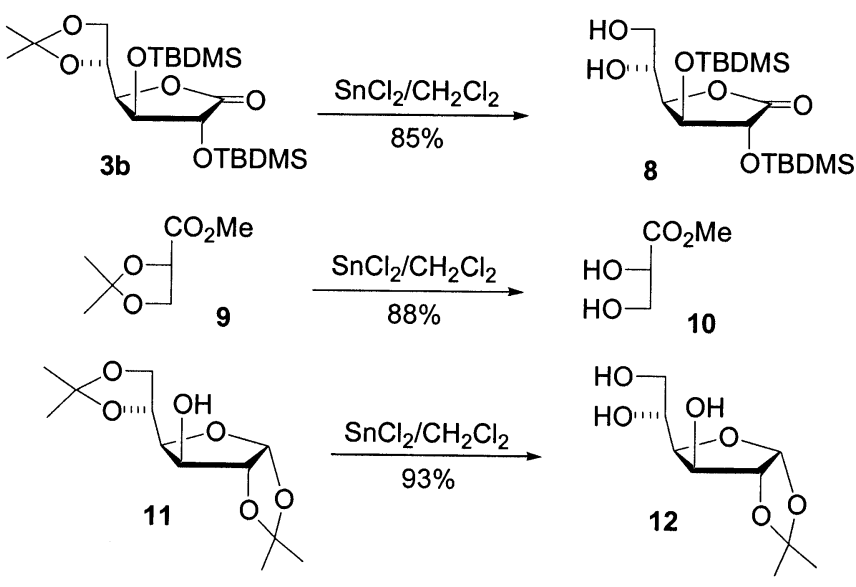

Figure 3. 

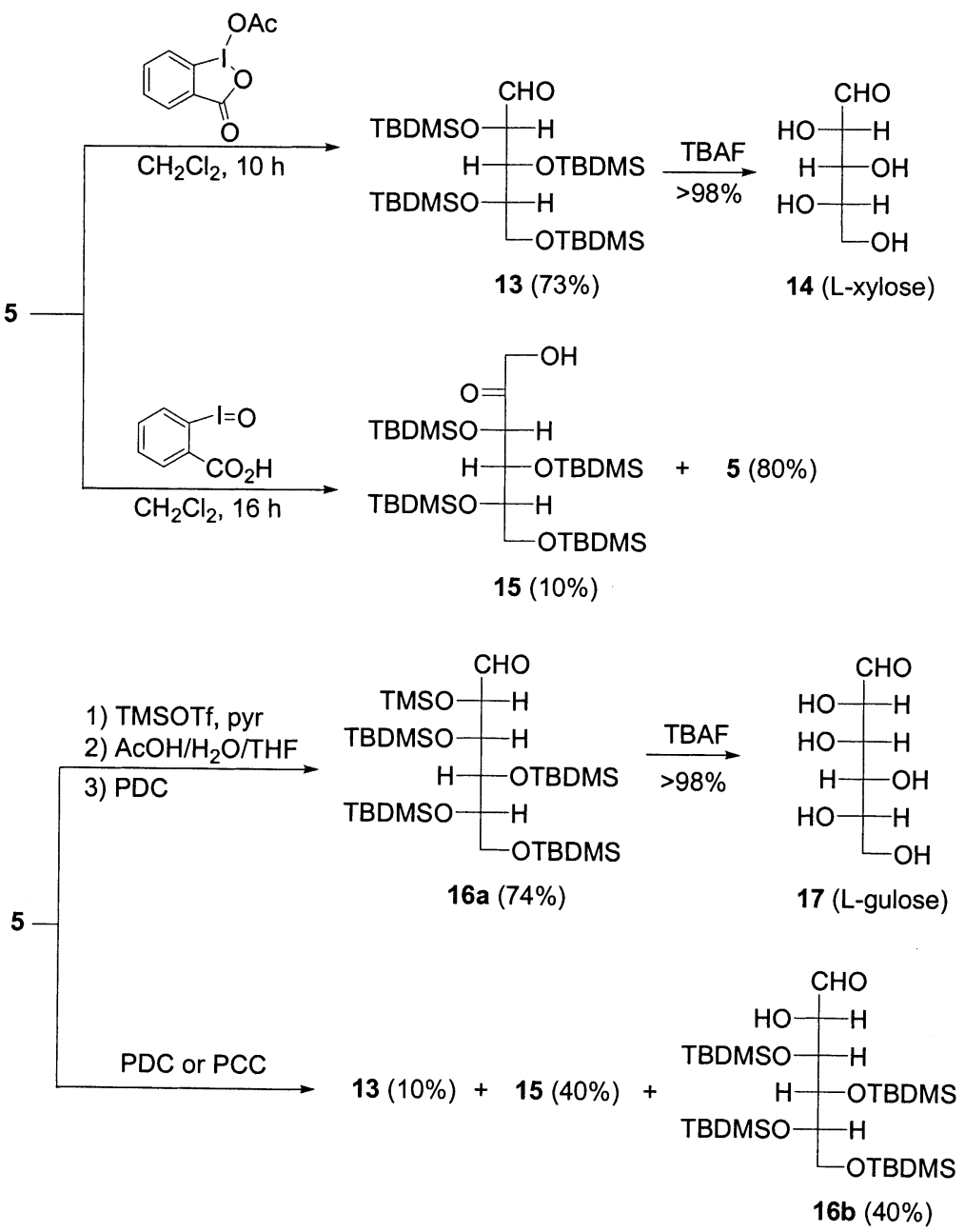

Scheme 2.

yield. Removal of the TBDMS groups resulted in a levorotatory product $14,[\alpha]_{\mathrm{D}}{ }^{24}=-19.8^{\circ}\left(c 5.0, \mathrm{H}_{2} \mathrm{O}\right)$, which was determined to be L-xylose by comparison of its ${ }^{1} \mathrm{H}$ NMR spectrum with that of D-xylose. The reactivity of $o$-iodosobenzoic acid $(\mathrm{IBX})^{9}$ toward diol 5 was rather low. When diol 5 was stirred with IBX at $22^{\circ} \mathrm{C}$ for $16 \mathrm{~h}$, a $10 \%$ yield of hydroxy ketone $\mathbf{1 5}$ was obtained accompanied by an $80 \%$ recovery of the diol. The ketose $\mathbf{1 5}$ may serve as a precursor for the synthesis of L-ascorbic acid, providing that the $\mathrm{CH}_{2} \mathrm{OH}$ group can be elaborated to $\mathrm{CHO}$ or $\mathrm{COOH}$ groups. ${ }^{10}$ This investigation will be conducted in due course.

On the other hand, diol $\mathbf{5}$ was treated with trimethylsilyl (trifluoromethane)sulfonate (TMSOTf), followed by selective cleavage of the primary TMS ether under mild acidic conditions $\left(\mathrm{AcOH} / \mathrm{THF} / \mathrm{H}_{2} \mathrm{O}=1 / 4 / 1\right.$ at $0{ }^{\circ} \mathrm{C}$ for $\left.1 \mathrm{~h}\right){ }^{11}$ The subsequent oxidation with pyridinium dichromate (PDC) produced aldehyde 16a in $74 \%$ overall yield. Deprotection of compound $\mathbf{1 6} \mathbf{a}$ with tetrabutylammonium fluoride (TBAF) gave a quantitative yield of L-gulose having the optical rotation $[\alpha]_{\mathrm{D}}=+21.0^{\circ}\left(c 0.95, \mathrm{H}_{2} \mathrm{O}\right)$ in agreement with the literature value, ${ }^{12}[\alpha]_{\mathrm{D}}=+21.3^{\circ}\left(c 4.58, \mathrm{H}_{2} \mathrm{O}\right)$. The ${ }^{1} \mathrm{H}$ and ${ }^{13} \mathrm{C}$ NMR spectra of the synthetic sample were identical with those of authentic sample. When diol 5 was subjected to oxidation with PDC or pyridinium chlorochromate (PCC), compounds 13, 15 and 16b were obtained in 10,40 and $40 \%$ yields, respectively. ${ }^{13}$ Removal of the TBDMS groups in $\mathbf{1 6 b}$ also led to L-gulose.

Thus, our present method has the advantage of the built-in absolute configurations of D-gluconolactone to provide an efficient synthesis of L-gulose by inversion of the two terminal groups (at $\mathrm{C}_{1}$ and $\mathrm{C}_{6}$ ). ${ }^{14}$ The previous preparations $^{12,15}$ of L-gulose are less practical because these procedures often require creating new stereocenters by various reactions. In many cases, perfect stereoselectivity is hardly achieved.

\section{Conclusion}

In summary, pure L-xylose and L-gulose were prepared from D-gluconolactone in reasonable yields by relatively short sequences. The selective deprotection of acetonides, in the presence of esters and silyl ethers, was realized by using $\mathrm{SnCl}_{2}$ as the promoter. This finding is significant because it is generally applicable to carbohydrate chemistry. 


\section{Experimental}

\subsection{General}

Melting points are uncorrected. Optical rotations were measured on a digital polarimeter with a cuvette of $10 \mathrm{~cm}$ length. ${ }^{1} \mathrm{H}$ NMR spectra were recorded at 400 or $500 \mathrm{MHz}$ with $\mathrm{CHCl}_{3}\left(\delta_{\mathrm{H}} 7.24\right)$ or $\mathrm{CD}_{3} \mathrm{OD}\left(\delta_{\mathrm{H}} 3.30\right.$ (central line of quintet)) as the internal standard; ${ }^{13} \mathrm{C}$ NMR spectra were recorded at 100 or $125 \mathrm{MHz}$ with $\mathrm{CDCl}_{3}\left(\delta_{\mathrm{C}} 77.0\right.$ (central line of triplet)) or $\mathrm{CD}_{3} \mathrm{OD}\left(\delta_{\mathrm{C}} 49.0\right.$ (central line of septet)) as the internal standard. Mass spectra were recorded at an ionizing voltage of 70 or $20 \mathrm{eV}$. Column chromatography was performed on silica gel (70-230 mesh); gradients of EtOAc and hexanes as well as gradients of $\mathrm{MeOH}$ and $\mathrm{CHCl}_{3}$ were used as eluents.

4.1.1. 5,6-O-Isopropylidene-D-glucono-1,4-lactone (2). ${ }^{5}$ Finely powdered D-glucono- 1,5 -lactone $(1.78 \mathrm{~g}, 10 \mathrm{mmol})$ was dissolved in an anhydrous DMF solution (10 mL) containing 2,2-dimethoxypropane $(4.16 \mathrm{~g}, 4.91 \mathrm{~mL}, 40$ $\mathrm{mmol}$ ) and tin (II) chloride (300 $\mathrm{mg}, 1.6 \mathrm{mmol})$. The mixture was stirred at $40^{\circ} \mathrm{C}$ under $\mathrm{Ar}$ for $15 \mathrm{~h}$. The solvent was then removed under reduced pressure and the residue was purified by chromatography on a silica gel column with hexanes/EtOAc (2/1) to give the desired product (1.85 g, $85 \%$ yield) as a white solid. IR $\left(\mathrm{KBr}, \mathrm{cm}^{-1}\right) 3445$ (broad, $\mathrm{OH}), 1756,1369,1212,1067,992,847 ;{ }^{1} \mathrm{H}$ NMR (400 $\left.\mathrm{MHz}, \mathrm{CD}_{3} \mathrm{OD}\right) \delta 1.34\left(\mathrm{~s}, 3 \mathrm{H}, \mathrm{CH}_{3}\right), 1.41\left(\mathrm{~s}, 3 \mathrm{H}, \mathrm{CH}_{3}\right)$, $3.94(\mathrm{dd}, 1 \mathrm{H}, J=8.7,5.9 \mathrm{~Hz}, \mathrm{H}-6 \mathrm{a}), 4.05(\mathrm{~d}, 1 \mathrm{H}, J=$ $2.7 \mathrm{~Hz}, \mathrm{H}-2), 4.10$ (dd, 1H, J=8.7, $6.5 \mathrm{~Hz}, \mathrm{H}-6 \mathrm{~b}), 4.23$ (dd, $1 \mathrm{H}, J=4.4,2.7 \mathrm{~Hz}, \mathrm{H}-3$ ), 4.43 (ddd, $1 \mathrm{H}, J=6.5,6.1$, $5.9 \mathrm{~Hz}, \mathrm{H}-5), 4.62(\mathrm{dd}, 1 \mathrm{H}, J=6.1,4.4 \mathrm{~Hz}, \mathrm{H}-4), 4.85$ (br s, $2 \mathrm{H}, \mathrm{OH} \times 2) ;{ }^{13} \mathrm{C} \mathrm{NMR}\left(100 \mathrm{MHz}, \mathrm{CD}_{3} \mathrm{OD}\right) \delta 25.43,26.80$, $66.91,73.87,74.39,75.25,83.19,110.36,177.03$. MS FAB $\mathrm{m} / \mathrm{z}$ (rel. intensity): $219\left(\mathrm{M}+\mathrm{H}^{+}, 100\right), 203(78), 161$ (24), 115 (29). HRMS (FAB) calcd for $\mathrm{C}_{9} \mathrm{H}_{15} \mathrm{O}_{6}\left(\mathrm{M}+\mathrm{H}^{+}\right)$ 219.0869 , found 219.0858 .

4.1.2. 2,3-Di-O-tert-butyldimethylsilyl-5,6-O-isopropylidene-D-glucono-1,4-lactone (3a). To a stirred solution of compound $2(360 \mathrm{mg}, 1.3 \mathrm{mmol})$ in anhydrous DMF ( $5 \mathrm{~mL})$ at room temperature $\left(20^{\circ} \mathrm{C}\right)$ were added pyridine $(2.7 \mathrm{~mL}$, $36 \mathrm{mmol}$ ) and tert-butyldimethylsilyl trifluoromethanesulfonate (TBDMSOTf, $785 \mathrm{mg}, 0.68 \mathrm{~mL}, 2.6 \mathrm{mmol}$ ). The mixture was stirred at $20^{\circ} \mathrm{C}$ for $20 \mathrm{~h}$, and then evaporated under reduced pressure. The resulting residue was purified by silica gel chromatography with hexanes/EtOAc (5/1) to give $662 \mathrm{mg}$ of 3a in $92 \%$ yield as a white solid. Mp 43$44^{\circ} \mathrm{C} ;[\alpha]_{\mathrm{D}}{ }^{18}=+38.4^{\circ}\left(c\right.$ 5, $\left.\mathrm{CHCl}_{3}\right) ; R_{\mathrm{f}}=0.35$ (EtOAc/ hexanes=1/20); IR (KBr, cm $\left.{ }^{-1}\right) 1800,1472,1372,1257$, $1124,1073,1051,839,782 ;{ }^{1} \mathrm{H}$ NMR (400 MHz, $\left.\mathrm{CDCl}_{3}\right) \delta$ 0.09 (s, $\left.3 \mathrm{H}, \mathrm{CH}_{3}\right), 0.10$ (s, $\left.3 \mathrm{H}, \mathrm{CH}_{3}\right), 0.13\left(\mathrm{~s}, 3 \mathrm{H}, \mathrm{CH}_{3}\right), 0.14$ (s, $\left.3 \mathrm{H}, \mathrm{CH}_{3}\right), 0.86\left(\mathrm{~s}, 9 \mathrm{H}, \mathrm{C}\left(\mathrm{CH}_{3}\right)_{3}\right), 0.87\left(\mathrm{~s}, 9 \mathrm{H}, \mathrm{C}\left(\mathrm{CH}_{3}\right)_{3}\right)$, $1.32\left(\mathrm{~s}, 3 \mathrm{H}, \mathrm{CH}_{3}\right), 1.40\left(\mathrm{~s}, 3 \mathrm{H}, \mathrm{CH}_{3}\right), 3.94(\mathrm{~d}, 1 \mathrm{H}, J=1.4 \mathrm{~Hz}$, $\mathrm{H}-2), 3.96$ (dd, $1 \mathrm{H}, J=8.8,5.5 \mathrm{~Hz}, \mathrm{H}-6 \mathrm{a}), 4.11(\mathrm{dd}, 1 \mathrm{H}$, $J=8.8,6.0 \mathrm{~Hz}, \mathrm{H}-6 \mathrm{~b}$ ), 4.12 (dd, $1 \mathrm{H}, J=3.2,1.4 \mathrm{~Hz}, \mathrm{H}-3$ ), $4.31(\mathrm{ddd}, 1 \mathrm{H}, J=8.2,6.0,5.5 \mathrm{~Hz}, \mathrm{H}-5), 4.46(\mathrm{dd}, 1 \mathrm{H}$, $J=8.2,3.2 \mathrm{~Hz}, \mathrm{H}-4) ;{ }^{13} \mathrm{C}$ NMR $\left(100 \mathrm{MHz}, \mathrm{CDCl}_{3}\right) \delta$ $-5.16(2 \times),-4.89,-4.81,18.03,18.05,25.21,25.54$, 25.60, 26.76, 67.13, 71.67, 74.40, 75.28, 82.93, 109.44, 174.03; FAB-MS $\mathrm{m} / \mathrm{z}$ (rel. intensity): $447\left(\mathrm{M}+\mathrm{H}^{+}, 8\right)$, 431 (18), 389 (27), 331 (16), 74 (100); HRMS (FAB) calcd for $\mathrm{C}_{21} \mathrm{H}_{43} \mathrm{O}_{6} \mathrm{Si}_{2} \quad\left(\mathrm{M}+\mathrm{H}^{+}\right)$447.2598, found 447.2605.

4.1.3. 1,2,3,4-Tetra-O-tert-butyldimethylsilyl-5,6-O-isopropylidene-D-glucitol (4). To a solution of compound 3a (500 mg, $1.12 \mathrm{mmol})$ in $\mathrm{THF} / \mathrm{H}_{2} \mathrm{O}(10 \mathrm{~mL}, 9 / 1)$ was added sodium borohydride ( $200 \mathrm{mg}, 5.2 \mathrm{mmol})$. After stirring at room temperature for $2 \mathrm{~h}$, the reaction was quenched by addition of acetic acid $(2 \mathrm{~mL})$. The reaction mixture was stirred for an additional $30 \mathrm{~min}$, and extracted with EtOAc $(20 \mathrm{~mL} \times 3)$. The collected organic layers were dried over anhydrous $\mathrm{Na}_{2} \mathrm{SO}_{4}$ and evaporated in vacuo. The resulting residue was purified by chromatography on a silica gel column with hexanes/EtOAc (5/1) to give the diol product (504 mg) in a quantitative yield. ESI-MS $\left(\mathrm{C}_{21} \mathrm{H}_{46} \mathrm{O}_{6} \mathrm{Si}_{2}\right) \mathrm{m} / \mathrm{z}$ (rel. intensity) $451\left(\mathrm{M}+\mathrm{H}^{+}, 100\right), 411(31), 393\left([\mathrm{M}-57]^{+}\right.$, 90).

To a solution of the above product $(500 \mathrm{mg}, 1.11 \mathrm{mmol}$ ) in anhydrous $\mathrm{CH}_{2} \mathrm{Cl}_{2}(15 \mathrm{~mL})$ were added TBDMSOTf $(722 \mathrm{mg}, 0.7 \mathrm{~mL}, 6.6 \mathrm{mmol})$ and pyridine $(1.86 \mathrm{~g}, 2.3 \mathrm{~mL}$, $22.2 \mathrm{mmol})$ at room temperature $\left(24^{\circ} \mathrm{C}\right)$. After stirring for $15 \mathrm{~h}$, the mixture was quenched with water, and extracted with EtOAc $(20 \mathrm{~mL} \times 3)$. The organic phase was washed with brine, dried over anhydrous $\mathrm{Na}_{2} \mathrm{SO}_{4}$, and concentrated under reduced pressure. The residue $(750 \mathrm{mg}$ ) was purified by silica gel chromatography with hexanes/EtOAc (100/1) to give $680 \mathrm{mg}$ of product $\mathbf{4}$ in $90 \%$ yield. $R_{\mathrm{f}}=0.75$ (EA/ hexanes $=1 / 5) ;[\alpha]_{\mathrm{D}}{ }^{20}=+10^{\circ}\left(c 1.2, \mathrm{CHCl}_{3}\right) ;{ }^{1} \mathrm{H}$ NMR $\left(400 \mathrm{MHz}, \mathrm{CDCl}_{3}\right) \delta 0.02\left(\mathrm{~s}, 6 \mathrm{H}, \mathrm{SiCH}_{3}\right), 0.03(\mathrm{~s}, 3 \mathrm{H}$, $\left.\mathrm{SiCH}_{3}\right), 0.06$ (s, 3H, $\left.\mathrm{SiCH}_{3}\right), 0.08$ (s, 6H, $\left.\mathrm{SiCH}_{3}\right), 0.09$ (s, $\left.3 \mathrm{H}, \mathrm{SiCH}_{3}\right), 0.10\left(\mathrm{~s}, 3 \mathrm{H}, \mathrm{SiCH}_{3}\right), 0.87\left(\mathrm{~s}, 9 \mathrm{H}, \mathrm{C}\left(\mathrm{CH}_{3}\right)_{3}\right), 0.88$ (s, $\left.18 \mathrm{H}, \mathrm{C}\left(\mathrm{CH}_{3}\right)_{3}\right), 0.90\left(\mathrm{~s}, 9 \mathrm{H}, \mathrm{C}\left(\mathrm{CH}_{3}\right)_{3}\right), 1.26\left(\mathrm{~s}, 3 \mathrm{H}, \mathrm{CH}_{3}\right)$, $1.36\left(\mathrm{~s}, 3 \mathrm{H}, \mathrm{CH}_{3}\right), 3.56$ (dd, $\left.1 \mathrm{H}, J=9.9,6.5 \mathrm{~Hz}, \mathrm{H}-6 \mathrm{a}\right), 3.73$ (dd, $1 \mathrm{H}, J=9.9,4.4 \mathrm{~Hz}, \mathrm{H}-6 \mathrm{~b}$ ), 3.80 (dd, $1 \mathrm{H}, J=7.5,7.2 \mathrm{~Hz}$, H-1a), 3.82 (dd, 1H, $J=6.6,3.6 \mathrm{~Hz}, \mathrm{H}-4), 3.85$ (dt, $1 \mathrm{H}, J=$ 6.6, $4.4 \mathrm{~Hz}, \mathrm{H}-5), 3.99$ (dd, 1H, J=7.5, 4.6 Hz, H-1b), 4.00 (dd, $1 \mathrm{H}, J=3.6,5.0 \mathrm{~Hz}, \mathrm{H}-3), 4.30$ (ddd, $1 \mathrm{H}, J=7.2$, $5.0,4.6 \mathrm{~Hz}, \mathrm{H}-2) ;{ }^{13} \mathrm{C}$ NMR $\left(100 \mathrm{MHz}, \mathrm{CDCl}_{3}\right) \delta-5.28$, $-5.17,-4.44,-4.32,-3.89,-3.70,-3.28,-3.17,18.19$, $18.30,18.40(2 \times), 24.97,26.02(2 \times), 26.06,26.14$, 26.47, 64.20, 66.48, 72.68, 73.08, 74.90, 75.42, 107.83 . $\mathrm{C}_{33} \mathrm{H}_{74} \mathrm{O}_{6} \mathrm{Si}_{4}$ ESI-MS $\mathrm{m} / z$ (rel. intensity): $679\left(\mathrm{M}+\mathrm{H}^{+}\right.$, 10), 677 (28), 563 (40), 447 (100), 391 (54).

4.1.4. 1,2,3,4-Tetra-O-tert-butyldimethylsilyl-D-glucitol (5). Compound 4 (100 mg, $0.15 \mathrm{mmol})$ and tin(II) chloride (70 mg, $0.37 \mathrm{mmol}$ ) were mixed in anhydrous $\mathrm{CH}_{2} \mathrm{Cl}_{2}$ $(5 \mathrm{~mL})$ and stirred at room temperature for $0.5 \mathrm{~h}$. The reaction progress was monitored by TLC with hexanes/ EtOAc (5/1). The undissolved tin chloride was removed by filtration, and the filtrate was neutralized with saturated $\mathrm{NaHCO}_{3}$ solution. The organic layer was dried over anhydrous $\mathrm{Na}_{2} \mathrm{SO}_{4}$, and concentrated. The resulting residue was subjected to silica gel chromatography with hexanes/ EtOAc (10/1) to give $88 \mathrm{mg}$ of diol 5 in $92 \%$ yield as a white solid. $R_{\mathrm{f}}=0.7($ EtOAc/hexanes $=1 / 4), \mathrm{mp} 82-83^{\circ} \mathrm{C} ;[\alpha]_{\mathrm{D}}{ }^{20}=$ $+31.3^{\circ}\left(c 1, \mathrm{CHCl}_{3}\right)$; IR $\left(\mathrm{KBr}, \mathrm{cm}^{-1}\right) 3508$ (broad, OH), $1472,1254,1084,836,777 ;{ }^{1} \mathrm{H}$ NMR $\left(500 \mathrm{MHz}, \mathrm{CDCl}_{3}\right) \delta$ 0.03 (s, 6H, SiCH 3 ), 0.06 (s, 3H, $\left.\mathrm{SiCH}_{3}\right), 0.09$ (s, 9H, $\left.\mathrm{SiCH}_{3}\right), 0.10$ (s, $\left.3 \mathrm{H}, \mathrm{SiCH}_{3}\right), 0.14$ (s, $\left.3 \mathrm{H}, \mathrm{SiCH}_{3}\right), 0.87$ (s, 9H, $\left.\mathrm{C}\left(\mathrm{CH}_{3}\right)_{3}\right), 0.88\left(\mathrm{~s}, 9 \mathrm{H}, \mathrm{C}\left(\mathrm{CH}_{3}\right)_{3}\right), 0.88\left(\mathrm{~s}, 9 \mathrm{H}, \mathrm{C}\left(\mathrm{CH}_{3}\right)_{3}\right)$, $0.90\left(\mathrm{~s}, 9 \mathrm{H}, \mathrm{C}\left(\mathrm{CH}_{3}\right)_{3}\right), 2.08(\mathrm{dd}, 1 \mathrm{H}, J=4.9,7.9 \mathrm{~Hz}, \mathrm{OH})$, 
3.47-3.63 (m, 3H, H-6a, H-6b, H-4), 3.66 (ddd, 1H, J= $11.0,4.9,3.0 \mathrm{~Hz}, \mathrm{H}-5), 3.76(\mathrm{dd}, 1 \mathrm{H}, J=9.2,4.5 \mathrm{~Hz}$, $\mathrm{H}-1 \mathrm{a}), 3.91$ (dt, 1H, J=9.2, $4.5 \mathrm{~Hz}, \mathrm{H}-2), 3.95$ (s, 1H, $\mathrm{OH}), 4.00(\mathrm{~d}, 1 \mathrm{H}, J=4.5 \mathrm{~Hz}, \mathrm{H}-1 \mathrm{~b}), 4.05(\mathrm{t}, 1 \mathrm{H}, J=$ $7.5 \mathrm{~Hz}, \mathrm{H}-3) ;{ }^{13} \mathrm{C}$ NMR $\left(125 \mathrm{MHz}, \mathrm{CDCl}_{3}\right) \delta-5.25$, $-5.25,-5.20,-5.09,-4.21,-3.91,-3.63,-3.63$, $17.82,18.06,18.20,18.47,25.74,25.85,25.89,26.08$, 62.79, 63.57, 69.36, 70.14, 73.09, 73.70; ESI-MS m/z (rel. intensity): $639\left(\mathrm{M}+\mathrm{H}^{+}, 100\right), 507$ (7); HRMS (FAB) calcd for $\mathrm{C}_{30} \mathrm{H}_{71} \mathrm{O}_{6} \mathrm{Si}_{4}\left(\mathrm{M}+\mathrm{H}^{+}\right)$639.4328, found 639.4329.

4.1.5. 2,3,4-Tri-O-tert-butyldimethylsilyl-D-glucitol (6). Boron trichloride $\left(0.1 \mathrm{~mL}\right.$ of $1 \mathrm{M}$ solution in $\left.\mathrm{CH}_{2} \mathrm{Cl}_{2}\right)$ was added to a $\mathrm{CH}_{2} \mathrm{Cl}_{2}$ solution $(5 \mathrm{~mL})$ of compound $4(100 \mathrm{mg}$, $0.15 \mathrm{mmol})$ at $-78^{\circ} \mathrm{C}$. The reaction progress was monitored by TLC with hexanes/EtOAc (5/1). The reaction was stirred at $-78^{\circ} \mathrm{C}$ for $5 \mathrm{~min}$, after which the reaction mixture was neutralized with saturated $\mathrm{NaHCO}_{3}$ solution. The resulting mixture was extracted with $\mathrm{CH}_{2} \mathrm{Cl}_{2}$, dried over anhydrous $\mathrm{Na}_{2} \mathrm{SO}_{4}$, and concentrated. The residue was subjected to silica gel chromatography with hexanes/EtOAc (5/1) to give $67 \mathrm{mg}$ of diol 5 in $70 \%$ yield and $17 \mathrm{mg}$ of triol $\mathbf{6}$ in $20 \%$ yield. Compound 6: $R_{\mathrm{f}}=0.45($ EtOAc/hexanes $=1 / 4)$; ${ }^{1} \mathrm{H}$ NMR $\left(400 \mathrm{MHz}, \mathrm{CDCl}_{3}\right) \delta 0.05\left(\mathrm{~s}, 3 \mathrm{H}, \mathrm{SiCH}_{3}\right), 0.05(\mathrm{~s}$, $3 \mathrm{H}, \mathrm{SiCH}_{3}$ ), 0.09 (s, 9H, $\left.\mathrm{SiCH}_{3}\right), 0.10$ (s, 3H, $\left.\mathrm{SiCH}_{3}\right), 0.11$ (s, 3H, $\left.\mathrm{SiCH}_{3}\right), 0.12$ (s, 3H, $\left.\mathrm{SiCH}_{3}\right), 0.88$ (s, 9H, C( $\left.\left.\mathrm{CH}_{3}\right)_{3}\right)$, 0.89 (s, 9H, C( $\left.\left(\mathrm{CH}_{3}\right)_{3}\right), 0.90\left(\mathrm{~s}, 9 \mathrm{H}, \mathrm{C}\left(\mathrm{CH}_{3}\right)_{3}\right), 2.29$ (br s, $1 \mathrm{H}$, $\mathrm{OH}), 3.03$ (br s, 1H, OH), 3.47 (br s, $1 \mathrm{H}, \mathrm{OH}), 3.56(\mathrm{dd}, 1 \mathrm{H}$, $J=10.2,4.7 \mathrm{~Hz}, \mathrm{H}-1 \mathrm{a}), 3.63$ (dd, $1 \mathrm{H}, J=11.2,5.5 \mathrm{~Hz}, \mathrm{H}-6 \mathrm{a})$, 3.65 (dd, $1 \mathrm{H}, J=11.2,3.7 \mathrm{~Hz}, \mathrm{H}-1 \mathrm{~b}), 3.70$ (dd, $1 \mathrm{H}, J=8.7$, $5.6 \mathrm{~Hz}, \mathrm{H}-3), 3.76$ (dd, $1 \mathrm{H}, J=10.2,5.6 \mathrm{~Hz}, \mathrm{H}-1 \mathrm{~b}), 3.80$ (m, $1 \mathrm{H}, \mathrm{H}-5), 3.88$ (t, 1H, $J=5.6 \mathrm{~Hz}, \mathrm{H}-4), 3.93$ (ddd, $1 \mathrm{H}, J=$ 8.7, 5.6, $4.7 \mathrm{~Hz}, \mathrm{H}-2)$; ${ }^{13} \mathrm{C} \mathrm{NMR}\left(100 \mathrm{MHz}, \mathrm{CDCl}_{3}\right) \delta$ $-5.52,-5.43,-4.68,-4.62,-4.26,-3.98, \quad 18.12$, $18.18,18.30,25.85,25.91,25.93,63.60,65.39,71.79$, 72.83, 73.04, 73.82; ESI-MS $\mathrm{m} / \mathrm{z}$ (rel. intensity): 525.2 $\left(\mathrm{M}+\mathrm{H}^{+}, 100\right), 393.2$ (5); HRMS (FAB) calcd for $\mathrm{C}_{24} \mathrm{H}_{57} \mathrm{O}_{6} \mathrm{Si}_{3}\left(\mathrm{M}+\mathrm{H}^{+}\right)$525.3463, found 525.3485.

4.1.6. Methyl 2,3:5,6-di- $O$-isopropylidene-D-gluconate (7a) ${ }^{16}$ and methyl 3,4:5,6-di- $\boldsymbol{O}$-isopropylidene-D-gluconate (7b). ${ }^{17}$ As previously mentioned in the main text, compounds $\mathbf{7 a}$ and $\mathbf{7 b}$ were obtained as the side products in the reaction of D-glucono-1,5-lactone with 2,2-dimethoxypropane and tin(II) chloride. Compound 7a: ${ }^{1} \mathrm{H}$ NMR $\left(400 \mathrm{MHz}, \mathrm{CDCl}_{3}\right) \delta 1.32\left(\mathrm{~s}, 3 \mathrm{H}, \mathrm{CH}_{3}\right), 1.38(\mathrm{~s}, 3 \mathrm{H}$, $\left.\mathrm{CH}_{3}\right), 1.43\left(\mathrm{~s}, 3 \mathrm{H}, \mathrm{CH}_{3}\right), 1.46\left(\mathrm{~s}, 3 \mathrm{H}, \mathrm{CH}_{3}\right), 2.15(\mathrm{~d}, J=$ $9.4 \mathrm{~Hz}, 1 \mathrm{H}, \mathrm{OH}), 3.63$ (ddd, $J=9.4,8.5,1.9 \mathrm{~Hz}, 1 \mathrm{H}, \mathrm{H}-4)$, $3.76(\mathrm{~s}, 3 \mathrm{H}, \mathrm{OMe}), 4.03-3.98(\mathrm{~m}, 1 \mathrm{H}, \mathrm{H}-5), 4.10-4.05(\mathrm{~m}$, 2H, H-6), 4.39 (dd, J=1.9, $7.7 \mathrm{~Hz}, 1 \mathrm{H}, \mathrm{H}-3), 4.53$ (d, $J=7.7 \mathrm{~Hz}, 1 \mathrm{H}, \mathrm{H}-2)$. Compound $7 \mathbf{b}:{ }^{1} \mathrm{H}$ NMR $(400 \mathrm{MHz}$, $\left.\mathrm{CDCl}_{3}\right) \delta 1.32\left(\mathrm{~s}, 3 \mathrm{H}, \mathrm{CH}_{3}\right), 1.33\left(\mathrm{~s}, 3 \mathrm{H}, \mathrm{CH}_{3}\right), 1.36(\mathrm{~s}, 3 \mathrm{H}$, $\left.\mathrm{CH}_{3}\right), 1.40\left(\mathrm{~s}, 3 \mathrm{H}, \mathrm{CH}_{3}\right), 2.99(\mathrm{~d}, J=9.1 \mathrm{~Hz}, 1 \mathrm{H}, \mathrm{OH}), 3.81$ (s, 3H, OMe), 3.96 (dd, J=3.9, 8.2 Hz, 1H, H-6), 4.03 (d, $1 \mathrm{H}, J=7.5 \mathrm{~Hz}, 1 \mathrm{H}, \mathrm{H}-2), 4.06$ (ddd, $J=3.9,5.6,9.0 \mathrm{~Hz}, 1 \mathrm{H}$, H-5), 4.12 (dd, $J=5.6,8.2 \mathrm{~Hz}, 1 \mathrm{H}, \mathrm{H}-6), 4.19$ (dd, $J=1.5$, $7.5 \mathrm{~Hz}, 1 \mathrm{H}, \mathrm{H}-3), 4.31$ (dd, $J=1.5,9.0 \mathrm{~Hz}, 1 \mathrm{H}, \mathrm{H}-4) ;{ }^{13} \mathrm{C}$ NMR $\left(100 \mathrm{MHz}, \mathrm{CHCl}_{3}\right) \delta 25.25,26.51,26.65,27.14$, 52.64, 67.87, 69.44, 76.48, 77.27, 80.89, 109.85, 110.06, 172.97 .

4.1.7. 3,4:5,6-Di- $O$-isopropylidene-D-glucuronic acid (7c) and $1,2: 3,4: 5,6$-tri- $O$-isopropylidene-D-gluconate $(7 \mathrm{~d}){ }^{18}$
Finely powdered D-glucono-1,5-lactone (1.78 g, 10.0 $\mathrm{mmol})$ was dissolved in an acetone solution $(50 \mathrm{~mL})$ containing $\mathrm{I}_{2}(507.6 \mathrm{mg}, 2.0 \mathrm{mmol})$. The reaction was stirred at $45^{\circ} \mathrm{C}$ for $24 \mathrm{~h}$. The solvent was removed under reduced pressure to give a residue, which was chromatographed by a silica gel column with EtOAc/hexanes (1/1) to give a mixture of compounds $\mathbf{7 c}$ and $\mathbf{7 d}$. Compound $\mathbf{7 c}$ was crystallized, and its structure was confirmed by an $\mathrm{X}$-ray diffraction analysis. The mother liquor was further subjected to chromatography on a silica gel column $($ EtOAc/hexanes $=1 / 3)$ to afford pure compounds $7 \mathbf{c}$ and 7d. Compound 7c: ${ }^{1} \mathrm{H}$ NMR $\left(400 \mathrm{MHz}, \mathrm{CDCl}_{3}\right) \delta 1.33(\mathrm{~s}$, $\left.3 \mathrm{H}, \mathrm{CH}_{3}\right), 1.37\left(\mathrm{~s}, 3 \mathrm{H}, \mathrm{CH}_{3}\right), 1.40\left(\mathrm{~s}, 3 \mathrm{H}, \mathrm{CH}_{3}\right), 1.42(\mathrm{~s}, 3 \mathrm{H}$, $\left.\mathrm{CH}_{3}\right), 3.98(\mathrm{dd}, J=3.7,8.0 \mathrm{~Hz}, 1 \mathrm{H}, \mathrm{H}-6), 4.01$ (dd, $J=7.9$, $8.0 \mathrm{~Hz}, 1 \mathrm{H}, \mathrm{H}-4), 4.09$ (ddd, $J=2.1,3.7,8.0 \mathrm{~Hz}, 1 \mathrm{H}, \mathrm{H}-5$ ), $4.13(\mathrm{dd}, J=2.1,8.0 \mathrm{~Hz}, 1 \mathrm{H}, \mathrm{H}-6), 4.29$ (dd, $J=1.4,7.9 \mathrm{~Hz}$, $1 \mathrm{H}, \mathrm{H}-3), 4.40$ (d, J=1.4 Hz, 1H, H-2). Crystallographic data of 7c have been deposited with the Cambridge Crystallographic Data Center as the deposition number CCDC 173783. Copies of the data can be obtained, free of charge, on application to CCDC, 12 Union Road, Cambridge, CB2 1EZ, UK (fax: +44(0)-1223-336033 or e-mail: deposit@ ccdc.cam.ac.uk). Compound 7d: ${ }^{1} \mathrm{H}$ NMR $(400 \mathrm{MHz}$, $\left.\mathrm{CDCl}_{3}\right) \delta 1.31\left(\mathrm{~s}, 3 \mathrm{H}, \mathrm{CH}_{3}\right), 1.37\left(\mathrm{~s}, 3 \mathrm{H}, \mathrm{CH}_{3}\right), 1.37(\mathrm{~s}$, $\left.3 \mathrm{H}, \mathrm{CH}_{3}\right), 1.39\left(\mathrm{~s}, 3 \mathrm{H}, \mathrm{CH}_{3}\right), 1.54\left(\mathrm{~s}, 3 \mathrm{H}, \mathrm{CH}_{3}\right), 1.63$ (s, $\left.3 \mathrm{H}, \mathrm{CH}_{3}\right), 3.92(\mathrm{t}, J=8.4 \mathrm{~Hz}, 1 \mathrm{H}, \mathrm{H}-4), 3.96$ (dd, $J=3.9$, $8.2 \mathrm{~Hz}, 1 \mathrm{H}, \mathrm{H}-6), 4.07$ (ddd, $J=3.9,6.0,8.4 \mathrm{~Hz}, 1 \mathrm{H}, \mathrm{H}-5$ ), $4.12(\mathrm{dd}, J=6.0,8.2 \mathrm{~Hz}, 1 \mathrm{H}, \mathrm{H}-6), 4.25$ (dd, $J=1.5,8.4 \mathrm{~Hz}$, $1 \mathrm{H}, \mathrm{H}-3), 4.60$ (d, $J=1.5 \mathrm{~Hz}, 1 \mathrm{H}, \mathrm{H}-2)$.

4.1.8. 2,3-Di-O-tert-butyldimethylsilyl-D-glucono-1,4-lactone $(8),{ }^{19}$ methyl $\mathrm{L}$-glycerate $(\mathbf{1 0}),{ }^{20}$ and $1,2-O$-isopropylidene- $\boldsymbol{\alpha}$-D-glucofuranose (12). ${ }^{21}$ Compound $\mathbf{3 b}(35.2 \mathrm{mg}$, $0.079 \mathrm{mmol})$ and tin(II) chloride $(70 \mathrm{mg}, 0.37 \mathrm{mmol})$ were mixed in anhydrous $\mathrm{CH}_{2} \mathrm{Cl}_{2}(2 \mathrm{~mL})$, and stirred at room temperature. The reaction progress was monitored by TLC with $\mathrm{CHCl}_{3} / \mathrm{MeOH}(5 / 1)$. After $48 \mathrm{~h}$, the mixture was evaporated and purified by silica gel chromatography with $\mathrm{CHCl}_{3} / \mathrm{MeOH}(5 / 1)$ to give $27.3 \mathrm{mg}$ of diol 8 in $85 \%$ yield as a white solid. $R_{\mathrm{f}}=0.6\left(\mathrm{CHCl}_{3} / \mathrm{MeOH}=5 / 1\right) ;[\alpha]_{\mathrm{D}}{ }^{25}=$ $+34.0^{\circ}\left(c\right.$ 0.51, $\left.\mathrm{CHCl}_{3}\right) ;{ }^{1} \mathrm{H} \mathrm{NMR}\left(400 \mathrm{MHz}, \mathrm{CDCl}_{3}\right) \delta$ 0.14 (s, 3H, $\left.\mathrm{SiCH}_{3}\right), 0.15\left(\mathrm{~s}, 3 \mathrm{H}, \mathrm{SiCH}_{3}\right), 0.15(\mathrm{~s}, 6 \mathrm{H}$, $\left.\mathrm{SiCH}_{3}\right), 0.89$ (s, 9H, C $\left.\left(\mathrm{CH}_{3}\right)_{3}\right), 0.90\left(\mathrm{~s}, 9 \mathrm{H}, \mathrm{C}\left(\mathrm{CH}_{3}\right)_{3}\right), 2.59$ $(\mathrm{d}, J=5.4 \mathrm{~Hz}, 1 \mathrm{H}, \mathrm{OH}), 3.76$ (dd, $J=4.9,11.3 \mathrm{~Hz}, 1 \mathrm{H}, \mathrm{H}-6)$, 3.87 (dd, $J=2.4,11.3 \mathrm{~Hz}, 1 \mathrm{H}, \mathrm{H}-6), 3.99$ (m, 1H, H-5), 4.09 (d, $J=2.9 \mathrm{~Hz}, 1 \mathrm{H}, \mathrm{H}-2), 4.30$ (dd, $J=2.9,4.0 \mathrm{~Hz}, 1 \mathrm{H}, \mathrm{H}-3)$, $4.50(\mathrm{dd}, J=4.0,8.9 \mathrm{~Hz}, 1 \mathrm{H}, \mathrm{H}-4) ;{ }^{13} \mathrm{C}$ NMR $(100 \mathrm{MHz}$, $\left.\mathrm{CDCl}_{3}\right) \delta-5.03,-5.01,-4.72,-4.65,17.96,18.08$, 25.59, 25.61, 63.71, 68.63, 74.70, 74.94, 79.88, 173.69. HRMS (FAB) calcd for $\mathrm{C}_{18} \mathrm{H}_{39} \mathrm{O}_{6} \mathrm{Si}_{2}\left(\mathrm{M}+\mathrm{H}^{+}\right)$407.2285, found 407.2282. By similar procedures, deprotection of compounds 9 and 11 gave compounds $\mathbf{1 0}$ and $\mathbf{1 2}$ in 88 and $93 \%$ yields, respectively. Compound 10: ${ }^{1} \mathrm{H}$ NMR $\left(400 \mathrm{MHz}, \mathrm{CDCl}_{3}\right) \delta 4.25(\mathrm{bs}, 1 \mathrm{H}), 3.96-3.77(\mathrm{~m}, 2 \mathrm{H})$, $3.76(\mathrm{~s}, 3 \mathrm{H},-\mathrm{OMe}), 3.34(\mathrm{bs}, 1 \mathrm{H},-\mathrm{OH}), 2.63(\mathrm{bs}, 1 \mathrm{H}$, $-\mathrm{OH}) ;{ }^{13} \mathrm{C} \mathrm{NMR}\left(100 \mathrm{MHz}, \mathrm{CDCl}_{3}\right) \delta$ 52.64, 64.01, 71.79, 173.41; ${ }^{13} \mathrm{C}$ NMR (100 MHz, CD $\left.\mathrm{CD}_{3} \mathrm{OD}\right) \delta$ 51.29, 63.72, 71.99, 173.29. FAB-MS calcd for $\mathrm{C}_{4} \mathrm{H}_{9} \mathrm{O}_{4}\left(\mathrm{M}+\mathrm{H}^{+}\right)$ 121.0, found 121.1. Compound 12: ${ }^{1} \mathrm{H}$ NMR (400 MHz, $\left.\mathrm{DMSO}_{-} \mathrm{d}_{6}\right) \delta 1.21\left(\mathrm{~s}, 3 \mathrm{H}, \mathrm{CH}_{3}\right), 1.36\left(\mathrm{~s}, 3 \mathrm{H}, \mathrm{CH}_{3}\right), 3.34$ (m, 1H, H-6), 3.55 (ddd, J=3.0, 5.4, $11.2 \mathrm{~Hz}, 1 \mathrm{H}, \mathrm{H}-6$ ), $3.68(\mathrm{~m}, 1 \mathrm{H}, \mathrm{H}-5), 3.82$ (dd, $J=2.4,8.5 \mathrm{~Hz}, 1 \mathrm{H}, \mathrm{H}-4)$, 4.02 (bs, 1H, H-3), 4.36 (d, J=3.6 Hz, 1H, H-2), 4.48 (m, 
$1 \mathrm{H}, \mathrm{OH}), 4.66(\mathrm{~d}, J=5.9 \mathrm{~Hz}, 1 \mathrm{H}, \mathrm{OH}), 5.16(\mathrm{~d}, J=4.6 \mathrm{~Hz}$, $1 \mathrm{H}, \mathrm{OH}), 5.78(\mathrm{~d}, J=3.6 \mathrm{~Hz}, 1 \mathrm{H}, \mathrm{H}-1) ;{ }^{1} \mathrm{H} \mathrm{NMR}(400 \mathrm{MHz}$, $\left.\mathrm{CD}_{3} \mathrm{OD}\right) \delta 1.33\left(\mathrm{~s}, 3 \mathrm{H}, \mathrm{CH}_{3}\right), 1.48\left(\mathrm{~s}, 3 \mathrm{H}, \mathrm{CH}_{3}\right), 3.63(\mathrm{dd}$, $J=6.0,11.5 \mathrm{~Hz}, 1 \mathrm{H}, \mathrm{H}-6), 3.79$ (dd, $J=3.1,11.5 \mathrm{~Hz}, 1 \mathrm{H}$, H-6), 3.93 (ddd, $J=3.1,6.0,8.3 \mathrm{~Hz} 1 \mathrm{H}, \mathrm{H}-5$ ), 4.05 (dd, $J=2.6,8.3 \mathrm{~Hz}, 1 \mathrm{H}, \mathrm{H}-4), 4.24(\mathrm{~d}, J=2.6 \mathrm{~Hz}, 1 \mathrm{H}, \mathrm{H}-3)$, $4.51(\mathrm{~d}, J=3.6 \mathrm{~Hz}, 1 \mathrm{H}, \mathrm{H}-2) 5.90(\mathrm{~d}, J=3.6 \mathrm{~Hz}, 1 \mathrm{H}, \mathrm{H}-1)$; ${ }^{13} \mathrm{C}$ NMR $\left(100 \mathrm{MHz}\right.$, DMSO-d $\left.\mathrm{d}_{6}\right) \delta 26.22,26.77,63.82$, $68.57,73.43,80.19,84.79,104.57,110.59$.

\subsection{9. $\quad 2,3,4,5$-Tetra- $O$-tert-butyldimethylsilyl-L-xylose} (13). Diol $5(186 \mathrm{mg}, 0.30 \mathrm{mmol})$ dissolved in anhydrous $\mathrm{CH}_{2} \mathrm{Cl}_{2}(20 \mathrm{~mL})$ was mixed with Dess-Martin periodinane $(247 \mathrm{mg}, 0.58 \mathrm{mmol})$ at room temperature. The reaction mixture was stirred for $10 \mathrm{~h}$. The TLC analysis (EtOAc/ hexanes=1/5) showed a new spot. The reaction was quenched with saturated $\mathrm{NaHCO}_{3}(2 \mathrm{~mL})$ to give a clear solution. After stirring for $5 \mathrm{~min}$, the mixture was extracted with $\mathrm{CH}_{2} \mathrm{Cl}_{2}$, washed with brine, and dried over anhydrous $\mathrm{Na}_{2} \mathrm{SO}_{4}$. The collected organic layers were evaporated under reduced pressure to give $310 \mathrm{mg}$ of crude product. Further purification on a silica gel column with EtOAc/ hexanes (1/40) afforded $136 \mathrm{mg}$ of the desired aldehyde 13 in $73 \%$ yield. The compound can also be obtained as a minor product from the PCC or PDC oxidation. $R_{\mathrm{f}}=0.7$ $($ EtOAc/hexanes $=1 / 5) ;[\alpha]_{\mathrm{D}}^{20}=+23.8^{\circ}\left(\right.$ c $\left.0.8, \mathrm{CHCl}_{3}\right)$; IR $\left(\mathrm{KBr}, \mathrm{cm}^{-1}\right) 1738,1730,1471,1361,1255,1148,1091$, $1005,836,777 ;{ }^{1} \mathrm{H}$ NMR $\left(500 \mathrm{MHz}, \mathrm{CDCl}_{3}\right) \delta-0.02$ (s, $\left.6 \mathrm{H}, \mathrm{SiCH}_{3}\right),-0.01\left(\mathrm{~s}, 3 \mathrm{H}, \mathrm{SiCH}_{3}\right), 0.02\left(\mathrm{~s}, 3 \mathrm{H}, \mathrm{SiCH}_{3}\right), 0.03$ (s, $\left.3 \mathrm{H}, \mathrm{SiCH}_{3}\right), 0.05$ (s, $\left.3 \mathrm{H}, \mathrm{SiCH}_{3}\right), 0.07$ (s, 3H, $\left.\mathrm{SiCH}_{3}\right)$, $0.08\left(\mathrm{~s}, 3 \mathrm{H}, \mathrm{SiCH}_{3}\right), 0.83\left(\mathrm{~s}, 9 \mathrm{H}, \mathrm{C}\left(\mathrm{CH}_{3}\right)_{3}\right), 0.86(\mathrm{~s}, 9 \mathrm{H}$, $\left.\mathrm{C}\left(\mathrm{CH}_{3}\right)_{3}\right), 0.88\left(\mathrm{~s}, 9 \mathrm{H}, \mathrm{C}\left(\mathrm{CH}_{3}\right)_{3}\right), 0.90\left(\mathrm{~s}, 9 \mathrm{H}, \mathrm{C}\left(\mathrm{CH}_{3}\right)_{3}\right)$, 3.52 (dd, $1 \mathrm{H}, J=9.9,5.6 \mathrm{~Hz}, \mathrm{H}-5 \mathrm{a}), 3.67$ (dd, $1 \mathrm{H}, J=9.9$, $7.3 \mathrm{~Hz}, \mathrm{H}-5 \mathrm{~b}$ ), 3.83 (ddd, $1 \mathrm{H}, J=7.3,5.6,2.6 \mathrm{~Hz}, \mathrm{H}-4), 3.99$ $(\mathrm{d}, 1 \mathrm{H}, J=5.4 \mathrm{~Hz}, \mathrm{H}-2), 4.06(\mathrm{dd}, 1 \mathrm{H}, J=5.4,2.6 \mathrm{~Hz}, \mathrm{H}-3)$, $9.77(\mathrm{~s}, 1 \mathrm{H}, \mathrm{CHO}) ;{ }^{13} \mathrm{C} \mathrm{NMR}\left(125 \mathrm{MHz}, \mathrm{CDCl}_{3}\right) \delta-5.34$, $-5.30,-5.22,-4.84,-4.62,-4.60,-3.88(2 \times), 18.02$ (2×), 18.18, 18.34, $25.74(2 \times), 25.92$ (2×), 62.59, 72.38, 73.89, 77.42, 202.24; ESI-MS $\mathrm{m} / \mathrm{z}$ (rel. intensity) 635 $\left(\mathrm{M}+\mathrm{H}^{+}, 4\right), 432$ (100); HRMS (FAB) calcd for $\mathrm{C}_{30} \mathrm{H}_{67} \mathrm{O}_{6} \mathrm{Si}_{4}\left(\mathrm{M}+\mathrm{H}^{+}\right)$635.4006, found 635.4015.

4.1.10. L-Xylose (14). A solution of compound 13 (63 mg, $0.1 \mathrm{mmol})$ in THF $(5 \mathrm{~mL})$ was treated with $0.5 \mathrm{~mL}$ $(0.5 \mathrm{mmol})$ of tetrabutylammonium fluoride $(1.0 \mathrm{M}$ in THF), and stirred at $0^{\circ} \mathrm{C}$ for $2 \mathrm{~h}$. The mixture was concentrated, and purified by silica gel chromatography $\left(\mathrm{CHCl}_{3} /\right.$ $\mathrm{MeOH}=5 / 1$ ) to give $15 \mathrm{mg}$ of compound $\mathbf{1 4}$ (>98\% yield). $[\alpha]_{\mathrm{D}}{ }^{24}=-19.8^{\circ}\left(c\right.$ 5.0, $\left.\mathrm{H}_{2} \mathrm{O}\right)$. Compound 14 exhibited the ${ }^{1} \mathrm{H}$ and ${ }^{13} \mathrm{C}$ NMR spectra compatible to that of D-xylose. ${ }^{22}$

4.1.11. 3,4,5,6-Tetra- $O$-tert-butyldimethylsilyl-L-xylohexulose (15) and 3,4,5,6-tetra-O-tert-butyldimethylsilylL-gulose (16b). A mixture of diol 5 (63 $\mathrm{mg}, 0.1 \mathrm{mmol})$, pyridinium dichromate $(119 \mathrm{mg}, 0.3 \mathrm{mmol})$ and Celite $(200 \mathrm{mg})$ in anhydrous $\mathrm{CH}_{2} \mathrm{Cl}_{2}(10 \mathrm{~mL})$ was stirred at $25^{\circ} \mathrm{C}$ for $20 \mathrm{~h}$. The reaction mixture was filtered through Pyrex 3G3 filter, and the filtrate was evaporated under reduced pressure. The residue was purified by silica gel chromatography $($ EtOAc/hexanes $=1 / 40)$ to afford aldopentose 13 (6 mg, 10\%), ketohexose $15(26 \mathrm{mg}, 40 \%)$ and aldohexose 16b (25 mg, 40\%). Products 15 and 16b were also obtained by Jones' oxidation of diol $\mathbf{5}$. Compound $\mathbf{1 5}$ :
$R_{\mathrm{f}}=0.6 \quad($ EtOAc/hexanes $=1 / 10) . \quad$ IR $\quad\left(\mathrm{KBr}, \mathrm{cm}^{-1}\right) 3508$ (broad-OH), 1720, 1473, 1257, 1088, 837, 778; ${ }^{1} \mathrm{H}$ NMR $\left(400 \mathrm{MHz}, \mathrm{CDCl}_{3}\right) \delta-0.03\left(\mathrm{~s}, 3 \mathrm{H}, \mathrm{SiCH}_{3}\right),-0.02(\mathrm{~s}, 3 \mathrm{H}$, $\mathrm{SiCH}_{3}$ ), 0.01 (s, 6H, $\mathrm{SiCH}_{3}$ ), 0.04 (s, 3H, $\left.\mathrm{SiCH}_{3}\right), 0.05$ (s, $\left.3 \mathrm{H}, \mathrm{SiCH}_{3}\right), 0.06$ (s, 3H, $\left.\mathrm{SiCH}_{3}\right), 0.08\left(\mathrm{~s}, 3 \mathrm{H}, \mathrm{SiCH}_{3}\right), 0.86$ (s, 9H, C $\left.\left(\mathrm{CH}_{3}\right)_{3}\right), 0.87\left(\mathrm{~s}, 9 \mathrm{H}, \mathrm{C}\left(\mathrm{CH}_{3}\right)_{3}\right), 0.89(\mathrm{~s}, 9 \mathrm{H}$, $\left.\mathrm{C}\left(\mathrm{CH}_{3}\right)_{3}\right), 0.94\left(\mathrm{~s}, 9 \mathrm{H}, \mathrm{C}\left(\mathrm{CH}_{3}\right)_{3}\right), 3.03(\mathrm{t}, 1 \mathrm{H}, J=4.8 \mathrm{~Hz}$, $\mathrm{OH}), 3.58(\mathrm{dd}, 1 \mathrm{H}, J=9.2,3.7 \mathrm{~Hz}, \mathrm{H}-6 \mathrm{a}), 3.83(\mathrm{dd}, 1 \mathrm{H}$, $J=9.2,3.9 \mathrm{~Hz}, \mathrm{H}-6 \mathrm{~b}), 3.84$ (ddd, $1 \mathrm{H}, J=4.3,3.9,3.7 \mathrm{~Hz}$, H-5), 3.90 (dd, $1 \mathrm{H}, J=4.3,3.1 \mathrm{~Hz}, \mathrm{H}-4), 4.30$ (dd, $1 \mathrm{H}, J=$ $19.7,4.8 \mathrm{~Hz}, \mathrm{H}-1 \mathrm{a}), 4.42(\mathrm{~d}, 1 \mathrm{H}, J=3.1 \mathrm{~Hz}, \mathrm{H}-3), 4.55(\mathrm{dd}$, $1 \mathrm{H}, J=19.7,4.8 \mathrm{~Hz}, \mathrm{H}-1 \mathrm{~b}) ;{ }^{13} \mathrm{C}$ NMR $\left(100 \mathrm{MHz}, \mathrm{CDCl}_{3}\right) \delta$ $-5.40,-5.18,-4.84(2 \times),-4.78,-4.70,-4.67,-4.39$, $17.98,18.02,18.16(2 \times), 25.83,25.94,25.96,26.01,63.78$, 67.91, 75.39, 75.64, 76.81, 212.01; ESI-MS $\mathrm{m} / \mathrm{z}$ (rel. intensity) $659.6\left(\mathrm{M}+\mathrm{Na}^{+}, 100\right), 637.4\left(\mathrm{M}+\mathrm{H}^{+}, 20\right), 505.4(25)$, 373.3 (45). Compound 16b: $R_{\mathrm{f}}=0.7$ (EtOAc/hexanes $=1 /$ 10); IR ( $\left.\mathrm{KBr}, \mathrm{cm}^{-1}\right) 3453$ (broad, OH), 1739.5, 1472, $1255,1094,836,776 ;{ }^{1} \mathrm{H}$ NMR $\left(400 \mathrm{MHz}, \mathrm{CDCl}_{3}\right) \delta 0.02$ (s, 6H, SiCH 3 ), 0.06 (s, 6H, $\mathrm{SiCH}_{3}$ ), 0.09 (s, 6H, $\mathrm{SiCH}_{3}$ ), 0.09 (s, 3H, $\left.\mathrm{SiCH}_{3}\right), 0.10$ (s, 3H, $\left.\mathrm{SiCH}_{3}\right), 0.86$ (s, 9H, $\left.\mathrm{C}\left(\mathrm{CH}_{3}\right)_{3}\right), 0.87\left(\mathrm{~s}, 18 \mathrm{H}, \mathrm{C}\left(\mathrm{CH}_{3}\right)_{3}\right), 0.90\left(\mathrm{~s}, 9 \mathrm{H}, \mathrm{C}\left(\mathrm{CH}_{3}\right)_{3}\right)$, $3.56(\mathrm{dd}, 1 \mathrm{H}, J=9.9,6.6 \mathrm{~Hz}, \mathrm{H}-6 \mathrm{a}), 3.73(\mathrm{dd}, 1 \mathrm{H}, J=9.9$, $4.7 \mathrm{~Hz}, \mathrm{H}-6 \mathrm{~b}), 3.80$ (dd, $1 \mathrm{H}, J=6.6,5.8 \mathrm{~Hz}, \mathrm{H}-3), 3.84$ (ddd, $1 \mathrm{H}, J=9.2,6.6,4.7 \mathrm{~Hz}, \mathrm{H}-5), 3.99$ (dd, $1 \mathrm{H}, J=9.2,6.6 \mathrm{~Hz}$, $\mathrm{H}-4), 4.30$ (dd, $1 \mathrm{H}, J=7.2,5.8 \mathrm{~Hz}, \mathrm{H}-2), 9.77$ (s, 1H, CHO); ${ }^{13} \mathrm{C}$ NMR $\left(125 \mathrm{MHz}, \mathrm{CDCl}_{3}\right) \delta-5.28,-5.17,-4.44$, $-4.32,-3.89,-3.69,-3.29,-3.17,18.00,18.13,18.27$, 18.40, 26.02 (2×), 26.06, 26.13, 64.19, 66.47, 72.68, 73.08, 75.42, 202.25. ESI-MS $\mathrm{m} / \mathrm{z}$ (rel. intensity): $637\left(\mathrm{M}+\mathrm{H}^{+}\right.$, 100), 619 (20), 565 (40), 507 (55), 415 (45), 373 (40).

4.1.12. Jones' oxidation of diol 5. Jones' reagent was freshly prepared by dissolving $\mathrm{CrO}_{3}(1.33 \mathrm{~g})$ in $\mathrm{H}_{2} \mathrm{SO}_{4} /$ $\mathrm{H}_{2} \mathrm{O}(1.5 \mathrm{~mL} / 5 \mathrm{~mL})$. An excess of Jones reagent $(0.035$ $\mathrm{mL})$ was added dropwise to a solution of diol $5(25 \mathrm{mg}$, $0.039 \mathrm{mmol})$ in acetone $(1 \mathrm{~mL})$ at $0^{\circ} \mathrm{C}$ until the orange color of Jones' reagent persisted. The reaction mixture was stirred at $0^{\circ} \mathrm{C}$ for $0.5 \mathrm{~h}$. Water $(2 \mathrm{~mL})$ was added, and the reaction mixture was extracted with $\mathrm{CH}_{2} \mathrm{Cl}_{2}$, washed with brine, and dried over anhydrous $\mathrm{Na}_{2} \mathrm{SO}_{4}$. The combined organic layers were evaporated under reduced pressure to give $23 \mathrm{mg}$ of crude product. Further purification on a silica gel column with EtOAc/hexanes (1/40) afforded the desired aldehyde $\mathbf{1 6 b}(7.5 \mathrm{mg}, 30 \%$ yield) and ketohexose 15 (13 mg, 50\% yield).

4.1.13. 3,4,5,6-Tetra- $O$-tert-butyldimethylsilyl-2-O-trimethylsilyl-L-gulose (16a). Diol 5 (64 mg, $0.1 \mathrm{mmol}$ ) was treated with TMSOTf $(89 \mathrm{mg}, 72 \mu \mathrm{L}, 0.4 \mathrm{mmol})$ and pyridine $(158 \mathrm{mg}, 162 \mu \mathrm{L}, 2.0 \mathrm{mmol})$ at $25^{\circ} \mathrm{C}$ for $15 \mathrm{~h}$ to give the corresponding bis(trimethylsilyl) ether. After purification, $78 \mathrm{mg}$ of di-TMS derivative was obtained in $99 \%$ yield. The primary TMS ether was selectively cleaved by treatment with $\mathrm{AcOH} / \mathrm{H}_{2} \mathrm{O} / \mathrm{THF}(1 \mathrm{~mL} / 1 \mathrm{~mL} / 4 \mathrm{~mL})$ at $0^{\circ} \mathrm{C}$ for $1 \mathrm{~h}$. The mixture was purified to give $44 \mathrm{mg}$ of the deprotected product $(78 \%$ yield). The resulting primary alcohol $(35.5 \mathrm{mg}, 0.05 \mathrm{mmol})$ was stirred with a mixture of pyridinium dichromate $(37.6 \mathrm{mg}, 0.1 \mathrm{mmol})$ and Celite (37.6 mg) in anhydrous $\mathrm{CH}_{2} \mathrm{Cl}_{2}(3 \mathrm{~mL})$ at $25^{\circ} \mathrm{C}$ for $20 \mathrm{~h}$. The solids were removed by filtration (Pyrex 3G3), and the filtrate was concentrated. The residue was purified by silica gel chromatography with hexanes/EtOAc (20/1) to 
give 16a (33 mg, 95\% yield) as a colorless syrup. The overall yield was $74 \%$ starting from diol 5. $R_{\mathrm{f}}=0.55$ (EtOAc/ hexanes $=1 / 20) ;{ }^{1} \mathrm{H}$ NMR $\left(500 \mathrm{MHz}, \mathrm{CDCl}_{3}\right) \delta 0.02(\mathrm{~s}, 3 \mathrm{H}$, $\mathrm{SiCH}_{3}$ ), 0.03 (s, 3H, $\mathrm{SiCH}_{3}$ ), 0.03 (s, 3H, $\mathrm{SiCH}_{3}$ ), 0.04 (s, $3 \mathrm{H}, \mathrm{SiCH}_{3}$ ), 0.06 (s, 3H, $\mathrm{SiCH}_{3}$ ), 0.07 (s, 3H, $\left.\mathrm{SiCH}_{3}\right), 0.08$ $\left(\mathrm{s}, 3 \mathrm{H}, \mathrm{SiCH}_{3}\right), 0.09$ (s, 9H, $\left.\mathrm{SiCH}_{3}\right), 0.10$ (s, 3H, $\mathrm{SiCH}_{3}$ ), $0.84\left(\mathrm{~s}, 9 \mathrm{H}, \mathrm{C}\left(\mathrm{CH}_{3}\right)_{3}\right), 0.85\left(\mathrm{~s}, 9 \mathrm{H}, \mathrm{C}\left(\mathrm{CH}_{3}\right)_{3}\right), 0.88(\mathrm{~s}, 9 \mathrm{H}$, $\left.\mathrm{C}\left(\mathrm{CH}_{3}\right)_{3}\right), 0.90\left(\mathrm{~s}, 9 \mathrm{H}, \mathrm{C}\left(\mathrm{CH}_{3}\right)_{3}\right), 3.56(\mathrm{dd}, 1 \mathrm{H}, J=9.8$, $6.8 \mathrm{~Hz}, \mathrm{H}-6 \mathrm{a}), 3.78$ (dd, $1 \mathrm{H}, J=9.8,4.8 \mathrm{~Hz}, \mathrm{H}-6 \mathrm{~b}), 3.80-$ 3.94 (m, 2H, H-3, H-4), 3.98 (ddd, 1H, J=6.8, 4.8, $2.2 \mathrm{~Hz}$, $\mathrm{H}-5), 4.65$ (s, 1H, H-2), 9.68 (s, 1H, CHO); ${ }^{13} \mathrm{C}$ NMR $\left(125 \mathrm{MHz}, \mathrm{CDCl}_{3}\right) \delta-5.41,-5.30,-4.54,-4.13(2 \times)$, $-4.03,-3.91,-3.46,0.28,18.18,18.32(3 \times), 25.98$, $26.02,26.09,26.16,63.98,73.14,75.36,78.35,78.43$, 202.52; ESI-MS $\mathrm{m} / \mathrm{z}$ (rel. intensity) $851\left(\mathrm{M}+\mathrm{H}^{+}, 5\right), 637$ (7), 531 (70), 494 (90).

4.1.14. L-Gulose (17). ${ }^{15 \mathrm{a}, \mathrm{h}}$ A solution of $\mathbf{1 6 a}(71 \mathrm{mg}$, $0.1 \mathrm{mmol})$ in THF $(5 \mathrm{~mL})$ was treated with $0.6 \mathrm{~mL}$ $(0.6 \mathrm{mmol})$ of tetrabutylammonium fluoride $(1.0 \mathrm{M}$ in $\mathrm{THF}$ ) at $0^{\circ} \mathrm{C}$ for $2 \mathrm{~h}$, by the procedure similar to that for $\mathrm{L}$-xylose, to give L-gulose in $>98 \%$ yield. The TBDMS groups of $\mathbf{1 6 \mathbf { b }}$ were also removed by a similar procedure to give L-gulose. Colorless amorphous solid. $[\alpha]_{\mathrm{D}}{ }^{23}=$ $+21.0^{\circ}\left(c \quad 0.9, \mathrm{H}_{2} \mathrm{O}\right) ;{ }^{1} \mathrm{H}$ NMR $\left(400 \mathrm{MHz}, \mathrm{D}_{2} \mathrm{O}\right) \delta 3.65$ (dt, $1 \mathrm{H}, J=8.3,2.5 \mathrm{~Hz}, \mathrm{H}-5), 3.74-3.79$ (m, 2H, H-6a, H-6b), 3.83 (m, 1H, H-3), 4.02 (dd, 1H, J=6.0, $2.5 \mathrm{~Hz}$, H-4), 4.09 (dd, $1 \mathrm{H}, J=6.0,2.5 \mathrm{~Hz}, \mathrm{H}-2), 4.90$ (dd, $1 \mathrm{H}$, $J=8.4,2.5 \mathrm{~Hz}, \mathrm{H}-1) ;{ }^{13} \mathrm{C} \mathrm{NMR}\left(100 \mathrm{MHz}, \mathrm{D}_{2} \mathrm{O}\right) \delta 60.56$, 68.64, 68.98, 70.72, 73.37, 93.39; HRMS (FAB) calcd for $\mathrm{C}_{6} \mathrm{H}_{13} \mathrm{O}_{6}$ 181.0712, found 181.0746.

\section{Acknowledgements}

We thank the financial supports from the National Science Council (NSC-90-2113-M-001-058), National Health Research Institute (NSC-90-2323-B-001-004), and the Heritage Prize of the Lee Foundation (for C. -H. Lin).

\section{References}

1. (a) Lewis, M. D.; Cha, J. K.; Kishi, Y. J. Am. Chem. Soc. 1982 , 104, 4976. (b) Cipolla, L.; Liguori, L.; Nicotra, F.; Torri, G.; Vismara, E. Chem. Commun. 1996, 1253. (c) Horneman, A. M.; Lundt, I. Tetrahedron 1997, 53, 6879. (d) Fleet, G. W. J. Sugar Lactones as Useful Starting Materials. In Antibiotics and Antiviral Compounds: Chemical Synthesis and Modifications; Krohn, K., Kirst, H. A., Eds.; VCH: Weinheim, 1993; p 333.

2. Lundt, I. In Glycoscience, Synthesis of Substrate Analogs and Mimetics; Driguez, H., Thiem, J., Eds.; Springer: Berlin, 1997; pp 117-156.

3. (a) Du, Y.; Linhardt, R. J.; Vlahov, I. R. Tetrahedron 1998, 54, 9913. (b) Postema, M. H. D. C-Glycoside Synthesis; CRC: Boca Raton, FL, 1995.

4. For a recent example, see: Takahashi, H.; Yoshinori, I.; Ikegami, S. J. Am. Chem. Soc. 2000, 122, 2995.

5. Chittenden, G. J. F. Trav. Chim. Pays-Bas 1988, 107, 455.

6. (a) Yang, W.-B.; Tsai, C.-H.; Lin, C.-H. Tetrahedron Lett. 2000, 41, 2569. (b) Yang, Y.-Y.; Yang, W.-B.; Teo, C.-F.; Lin, C.-H. Synlett 2000, 1634. (c) Yang, W.-B.; Chang,
C.-F.; Wang, S.-H.; Teo, C.-F.; Lin, C.-H. Tetrahedron Lett. 2001, 42, 4657.

7. (a) Csuk, R.; Hugener, M.; Vasella, A. Helv. Chim. Acta 1988, 71, 609. (b) de Souza, I. C. B. V.; da Silva, M. N.; Ferreira, V. F. Synlett 1998, 1339. (c) Colle, S.; Taillefumier, C.; Chapleur, Y.; Liebl, R.; Schmidt, A. Bioorg. Med. Chem. 1999, 7, 1049.

8. Dess, D. B.; Martin, J. C. J. Am. Chem. Soc. 1991, 113, 7277.

9. (a) Frigerio, M.; Santagostino, M.; Sputore, S.; Palmisano, G. J. Org. Chem. 1995, 60, 7272. (b) De Munari, S.; Frigerio, M.; Santagostino, M. J. Org. Chem. 1996, 61, 9272. (c) Frigerio, M.; Santagostino, M.; Sputore, S. Synlett 1997, 833.

10. (a) Moser, V.; Bendick, A. B. In Handbook of the Vitamins; Macklin, L. J., Dekker, M., Eds.; 2nd ed, Wiley-Interscience: New York, 1991; pp 195-232. (b) Crueger, W.; Crueger, A. Biotechnology: A Textbook of Industrial Microbiology; Sinauer: Sunderland, MA, 1990 p 299.

11. Hart, T. W.; Metcalfe, D. A.; Scheinmann, F. Chem. Commun. 1979, 156.

12. Evans, M. E.; Parrish, F. W. Carbohydr. Res. 1973, 28, 359.

13. Oxidation of diol $\mathbf{5}$ with Jones' reagent gave the $\mathrm{C}_{1}$ oxidation product $16 \mathrm{~b}(30 \%)$ and the $\mathrm{C}_{2}$ oxidation product $15(50 \%)$. Silylation of $\mathbf{1 6} \mathbf{b}$ with TMSOTf gave a product identical with $16 a$.

14. A similar strategy has been used for the synthesis of L-ribose and L-glucose. See: (a) Jung, M. E.; Xu, Y. Tetrahedron Lett. 1997, 38, 4199. (b) Hajko, J.; Liptak, A.; Pozsgay, V. Carbohydr. Res. 1999, 321, 116.

15. (a) Ko, S. Y.; Lee, A. W. M.; Masamune, S.; Reed, III, L. A.; Sharpless, K. B.; Walker, F. J. Tetrahedron 1990, 46, 245. (b) Dondoni, A.; Marra, A.; Massi, A. J. Org. Chem. 1997, 62, 6261. (c) Katano, K.; Chang, P.-I.; Millar, A.; Pozsgay, V.; Minster, D. K.; Ohgi, T.; Hecht, S. M. J. Org. Chem. 1985, 50, 5807. (d) Minster, D. K.; Hecht, S. M. J. Org. Chem. 1978, 43, 3988. (e) Oshitari, T.; Shibasaki, M.; Yoshizawa, T.; Tomita, M.; Takao, K.; Kobayashi, S. Tetrahedron 1997, 53, 10993. (f) Boger, D. L. J. Am. Chem. Soc. 1994, 116, 5647. (g) Gonzalez, F. S.; Baer, H. H. Carbohydr. Res. 1990, 202, 33. (h) Takeuchi, M.; Taniguchi, T.; Ogasawara, K. Synthesis 1999, 341.

16. de Souza, M. C. B. V.; de Silva, M. N.; Ferreira, V. F. Synlett 1998, 1339.

17. Csuk, R.; Hugener, M.; Vasella, A. Helv. Chim. Acta 1998, 71, 609.

18. Yadav, J. S.; Barma, D. K. Tetrahedron 1996, 52, 4457.

19. Krulle, T. M.; de la Fuente, C.; Pickering, L.; Aplin, R. T.; Tsitsanou, K. E.; Zographos, S. E.; Oikonomakis, N. G.; Nash, R. J.; Griffiths, R. C.; Fleet, G. W. J. Tetrahedron: Asymmetry 1997, 8, 3807.

20. (a) Mukaiyama, T.; Shiina, I.; Iwadare, H.; Saitoh, M.; Nishimura, T.; Ohkawa, N.; Nishimura, K.; Tani, Y.; Hasegawa, M.; Yamada, K.; Saitoh, K. Chem. Eur. J. 1999, 5, 121. (b) Roodsari, F. S.; Wu, D.; Pum, G. S.; Hajdu, J. J. Org. Chem. 1999, 64, 7727.

21. (a) Cioletti, A. G.; Alves, R. J.; Filho, J. D. deS.; Chaves, J. G.; Peado, M. A. F. Synth. Commun. 2000, 30, 2019. (b) Norrild, J. C.; Eggert, H. J. Am. Chem. Soc. 1995, 117, 1479.

22. (a) Ahmad, T.; Kenne, L.; Olsson, K.; Theander, O. Carbohydr. Res. 1995, 276, 309. (b) Feather, M. S.; Harris, J. F. Adv. Carbohydr. Chem. Biochem. 1973, 28, 161. (c) Lemieux, R. U.; Stevens, J. D. Can. J. Chem. 1966, 44, 249. 